\title{
Phytotoxicity and Longevity of \\ Twenty-two Preemergent Herbicides used on Three Species of Container- grown Palms
}

Timothy K. Broschat

Additional INDEX WORDS. Dypsislutescens, Phoenix roebelenii, Washingtonia robusta, weed control

Summary. Twenty-two preemergent herbicides were applied at their maximum labeled rates and twice those rates to determine their safety and effectiveness on areca palm [D ypsislutescens ( $H$. Wendl.) Beentje \& D ransf.], pygmy date palm (Phonix roebelenii $O$ 'B rien), and mexican fan palm (Washingtonia robusta $\mathbf{H}$. Wendl.). T wo products, dichlobenil and metolachlor showed consistent phytotoxicity on all three species. Several of the remaining products caused death of the apical meristem in mexican fan palms and reduced growth rates in pygmy date palms, but most caused little damage to areca palms. H erbicides applied as sprays generally remained effective for 2 to 4 months, whereas granular products, especially those containing oxyfluorfen plus another chemical, were effective for up to 8 months.

W

eed control is one of the most labor intensive and expensive aspects of container palm production. Sincepalms are often grown in open-sided shadehouses or in full sun on the

U niversity of Florida, Ft. L auderdale Research \& Education Center, 3205 College Avenue, Ft. Lauderdale, FL 33314.

Florida Agricultural Experiment Station journal series R-07184. The author wishes to thank Susan Thor for her assistance in this project. This work was funded in part by the IR-4 M inor U se Pesticide Project. The cost of publishing this paper was defrayed in part by the payment of page charges. U nder postal regulations, this paper therefore must be hereby marked advertise ment solely to indicate this fact. 
T able 1. Preemergent herbicides evaluated on three species of container-grown palms.

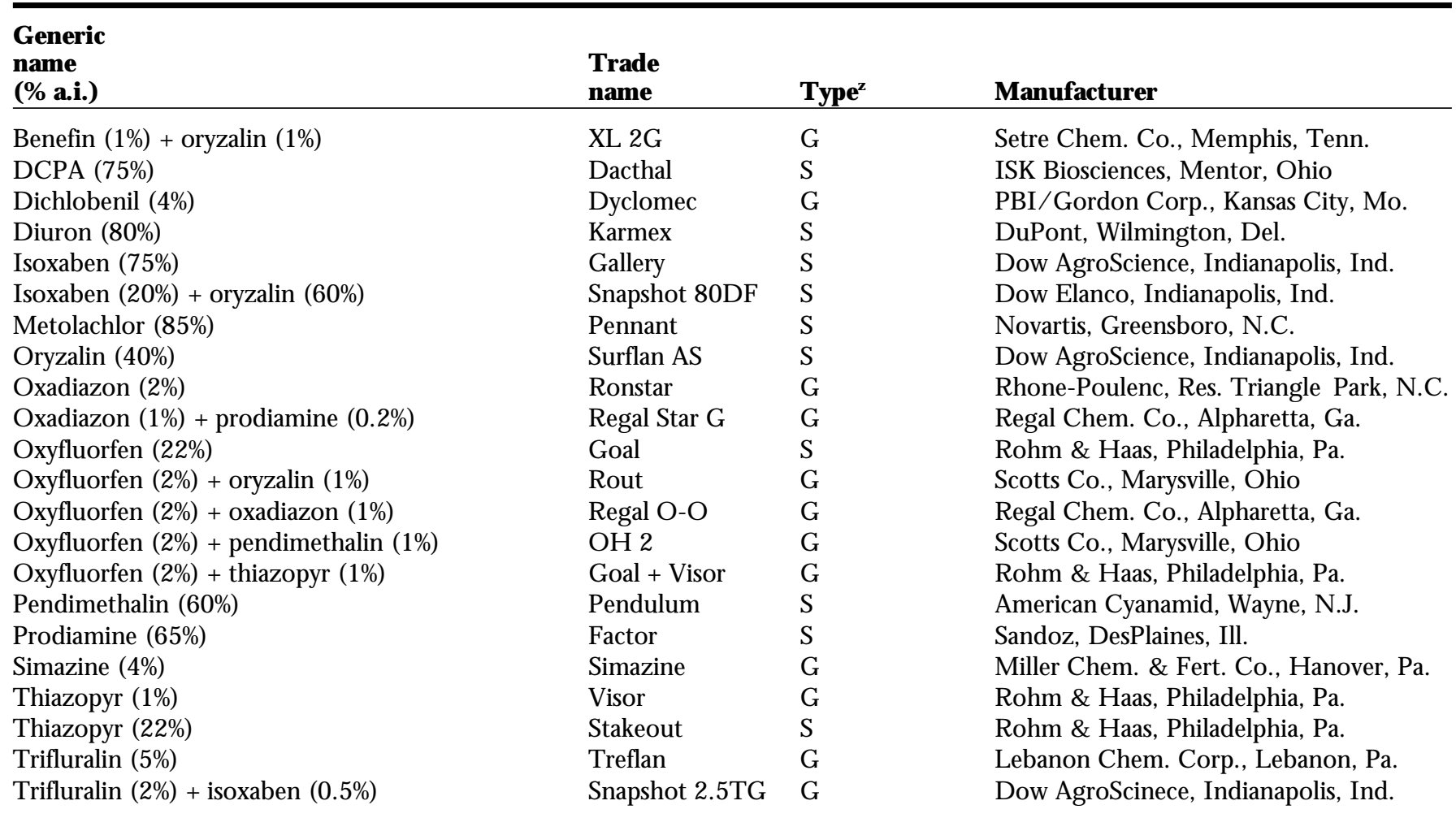

${ }^{\mathrm{z}} \mathrm{G}=$ granules, $\mathrm{S}=$ spray-applied.

ground over a long period of time, weed infestations are often more severe than they would be in plants growing on benches in a greenhouse. Theyear-round warm climate required for growing palms also results in yearround weed growth. Preemergent herbicideshave been widely used on other types of container-grown ornamental plants, but relatively few are labeled in the U nited States for use on palms. E arlier tests of some preemergent herbicides on several species of palms indicated that some of theproductstested were rather toxic to palms and that the symptoms of phytotoxicity on these slow-growing plants may not become apparent until 8 months or longer following herbicide application (Donselman and Broschat, 1986; $M$ eerow and Broschat, 1991). Since a number of temperate and subtropical weeds encountered during palm production are not readily controlled by some of the safer products currently labeled for palms (N eel, 1977), an experiment was set up to evaluate the phytotoxicity and efficacy of $22 \mathrm{com}$ mercially available preemergent herbicides that are currently labeled for use on palms or other types of containergrown ornamental plants.

\section{Materials and methods}

Seedlings of areca palm, pygmy date palm, and mexican fan palm growing in 1 qt $(1 \mathrm{~L})$ containers were potted up into 1 gal $(3.8 \mathrm{~L})$ plastic containers using a 5 pine bark: 4 sedge peat: 1 sand (by volume) substrate. This substrate was amended with dolomitic limestone at $12 \mathrm{lb} / \mathrm{yd}^{3}\left(7.1 \mathrm{~kg} \cdot \mathrm{m}^{-3}\right)$ and $\mathrm{M}$ icromax (Scotts Co., M arysville, O hio) at 1.5 $\mathrm{lb} / \mathrm{yd}^{3}\left(890 \mathrm{~g} \cdot \mathrm{m}^{-3}\right)$. All containers received a single dibbled application of O smocote $15 \mathrm{~N}-3.9 \mathrm{P}-10 \mathrm{~K}$ (ScottsC 0 .) and received about 0.8 inches $(2 \mathrm{~cm})$ of water daily from overhead irrigation and/ or rainfall. Plants were grown in a fabric-covered shadehouse (55\% light exclusion) and wereallowed to become infested naturally with weeds common in the nursery.

Eight replicate pots of each palm species were treated with 22 preemergent herbicides at their maximum label rate $(1 x)$ and twice that rate $(2 x)$ to determinetheir relativesafety on palms (Table 1). Granules were broadcast over the surface of the containers beneath the palm canopies. Foliar sprays were applied over the top of the foliage for all products except thiazopyr and oxyfluorfen, which were sprayed only over the soil surface as required by their labels. M etolachlor was applied over the foliage, but was immediately rinsed off with water according to its label. Foliar applied herbicides were applied in $5 \mathrm{fl}$ oz of spray solution over a $14 \mathrm{ft}^{2}$ area (150 mL over $1.3 \mathrm{~m}^{2}$ ) using a hand-held pump-up sprayer.

E very two monthsfor 10 months, all palms were rated subjectively on a 0 to 5 scale $(0=$ dead, $3=$ moderate injury, $5=$ no injury). The number of weeds of each weed species was also recorded for each pot with weeds being removed after each counting. Since weed numbers were relatively small for each individual weed species, their numbers were combined to determine herbicide efficacy. Plant shoot dry mass wasdetermined at theend of 10 months by cutting the palms at soil level and drying the tops at $140^{\circ} \mathrm{F}\left(60^{\circ} \mathrm{C}\right)$ until constant weight was achieved. D ata were analyzed by analysis of variance with mean separations by the WallerD uncan $\mathrm{k}$ ratio method.

\section{Results and discussion}

Рнyтотохісіту. A reca palms tolerated most of the 22 preemergent herbicides at both rates tested (Table 2 ). The most notable exception was 
T able 2. Phytotoxicity ratings and final shoot dry mass of areca pal ms treated with 22 preemergent herbicides. Data are means for eight replicate pots.

\begin{tabular}{|c|c|c|c|c|c|c|c|c|}
\hline \multirow[b]{2}{*}{ H erbicide } & \multirow{2}{*}{$\begin{array}{l}\text { Application } \\
\text { method }^{2}\end{array}$} & \multirow{2}{*}{$\begin{array}{c}\text { R ate } \\
\left(\text { a.i. lb/acre) }{ }^{w}\right. \\
\end{array}$} & \multicolumn{5}{|c|}{ Months after treatment } & \multirow{2}{*}{$\begin{array}{c}\text { Shoot } \\
\text { dry wt } \\
\text { (g)v }\end{array}$} \\
\hline & & & 2 & 4 & 6 & 8 & 10 & \\
\hline Control & & 0 & $5.0^{y} a^{x}$ & $5.0 \mathrm{a}$ & $5.0 \mathrm{a}$ & $5.0 \mathrm{a}$ & $5.0 \mathrm{a}$ & $118.0 \mathrm{a}-\mathrm{h}$ \\
\hline \multirow{2}{*}{ Benefin + oryzalin } & G & 6 & $5.0 \mathrm{a}$ & $4.9 a b$ & $5.0 \mathrm{a}$ & $5.0 \mathrm{a}$ & $5.0 \mathrm{a}$ & $125.5 \mathrm{a}-\mathrm{f}$ \\
\hline & & 12 & $4.9 \mathrm{a}$ & $5.0 \mathrm{a}$ & $4.9 a b$ & $5.0 \mathrm{a}$ & $5.0 \mathrm{a}$ & $112.6 \mathrm{~b}-\mathrm{i}$ \\
\hline \multirow[t]{2}{*}{ DCPA } & $\mathrm{S}$ & 10.5 & $5.0 \mathrm{a}$ & $5.0 \mathrm{a}$ & $5.0 \mathrm{a}$ & $5.0 \mathrm{a}$ & $5.0 \mathrm{a}$ & $144.9 a b$ \\
\hline & & 21.0 & $5.0 \mathrm{a}$ & $5.0 \mathrm{a}$ & $5.0 \mathrm{a}$ & $5.0 \mathrm{a}$ & $5.0 \mathrm{a}$ & 132.3 a-e \\
\hline \multirow[t]{2}{*}{ Dichlobenil } & G & 8 & $4.3 \mathrm{c}$ & $4.1 \mathrm{c}$ & $4.4 \mathrm{c}$ & $4.5 \mathrm{~b}$ & $5.0 \mathrm{a}$ & $81.0 \mathrm{i}-\mathrm{I}$ \\
\hline & & 16 & $3.9 \mathrm{~d}$ & $3.3 \mathrm{~d}$ & $2.8 \mathrm{~d}$ & $2.4 \mathrm{c}$ & $5.0 \mathrm{a}$ & 49.81 \\
\hline \multirow[t]{2}{*}{ Diuron } & $\mathrm{S}$ & 4 & $5.0 \mathrm{a}$ & $5.0 \mathrm{a}$ & $5.0 \mathrm{a}$ & $5.0 \mathrm{a}$ & $5.0 \mathrm{a}$ & $100.7 \mathrm{e}-\mathrm{j}$ \\
\hline & & 8 & $5.0 \mathrm{a}$ & $5.0 \mathrm{a}$ & $5.0 \mathrm{a}$ & $5.0 \mathrm{a}$ & $5.0 \mathrm{a}$ & $123.4 \mathrm{a}-\mathrm{g}$ \\
\hline \multirow[t]{2}{*}{ I soxaben } & $\mathrm{S}$ & 1 & $5.0 \mathrm{a}$ & $5.0 \mathrm{a}$ & $5.0 \mathrm{a}$ & $5.0 \mathrm{a}$ & $5.0 \mathrm{a}$ & $138.1 \mathrm{a}-\mathrm{c}$ \\
\hline & & 2 & $5.0 \mathrm{a}$ & $5.0 \mathrm{a}$ & $5.0 \mathrm{a}$ & $5.0 \mathrm{a}$ & $5.0 \mathrm{a}$ & $134.4 \mathrm{a}-\mathrm{d}$ \\
\hline \multirow[t]{2}{*}{ Isoxaben + oryzalin } & $\mathrm{S}$ & 4 & $5.0 \mathrm{a}$ & $5.0 \mathrm{a}$ & $5.0 \mathrm{a}$ & $5.0 \mathrm{a}$ & $5.0 \mathrm{a}$ & $111.8 \mathrm{~b}-\mathrm{i}$ \\
\hline & & 8 & $5.0 \mathrm{a}$ & $5.0 \mathrm{a}$ & $5.0 \mathrm{a}$ & $5.0 \mathrm{a}$ & $5.0 \mathrm{a}$ & $94.4 \mathrm{f}-\mathrm{j}$ \\
\hline \multirow[t]{2}{*}{ M etolachlor } & $\mathrm{S}$ & 8 & $5.0 \mathrm{a}$ & $5.0 \mathrm{a}$ & $4.8 \mathrm{~b}$ & $4.9 \mathrm{a}$ & $5.0 \mathrm{a}$ & $126.8 \mathrm{a}-\mathrm{f}$ \\
\hline & & 16 & $4.6 \mathrm{bc}$ & $4.6 a b$ & $4.8 \mathrm{~b}$ & $4.8 \mathrm{a}$ & $5.0 \mathrm{a}$ & $85.5 \mathrm{~h}-\mathrm{k}$ \\
\hline \multirow[t]{2}{*}{ Oryzalin } & S & 4 & $5.0 \mathrm{a}$ & $4.5 b c$ & $5.0 \mathrm{a}$ & $5.0 \mathrm{a}$ & $5.0 \mathrm{a}$ & $114.6 \mathrm{a}-\mathrm{h}$ \\
\hline & & 8 & $5.0 \mathrm{a}$ & $4.8 \mathrm{ab}$ & $5.0 \mathrm{a}$ & $5.0 \mathrm{a}$ & $5.0 \mathrm{a}$ & $127.3 \mathrm{a}-\mathrm{f}$ \\
\hline \multirow[t]{2}{*}{ Oxadiazon } & G & 4 & $5.0 \mathrm{a}$ & $5.0 \mathrm{a}$ & $5.0 \mathrm{a}$ & $5.0 \mathrm{a}$ & $5.0 \mathrm{a}$ & $99.6 \mathrm{e}-\mathrm{j}$ \\
\hline & & 8 & $5.0 \mathrm{a}$ & $5.0 \mathrm{a}$ & $5.0 \mathrm{a}$ & $5.0 \mathrm{a}$ & $5.0 \mathrm{a}$ & $107.2 \mathrm{c}-\mathrm{i}$ \\
\hline \multirow{2}{*}{\multicolumn{2}{|c|}{ Oxadiazon + prodiamine G }} & 2.4 & $5.0 \mathrm{a}$ & $5.0 \mathrm{a}$ & $5.0 \mathrm{a}$ & $5.0 \mathrm{a}$ & $5.0 \mathrm{a}$ & $124.7 \mathrm{a}-\mathrm{g}$ \\
\hline & & 4.8 & $5.0 \mathrm{a}$ & $5.0 \mathrm{a}$ & $5.0 \mathrm{a}$ & $5.0 \mathrm{a}$ & $5.0 \mathrm{a}$ & $120.6 \mathrm{a}-\mathrm{g}$ \\
\hline \multirow[t]{2}{*}{ Oxyflurofen } & $\mathrm{S}$ & 1 & $4.9 a$ & $4.9 \mathrm{ab}$ & $5.0 \mathrm{a}$ & $5.0 \mathrm{a}$ & $5.0 \mathrm{a}$ & $73.3 \mathrm{j}-\mathrm{I}$ \\
\hline & & 2 & $4.9 \mathrm{a}$ & $4.9 a b$ & $5.0 \mathrm{a}$ & $5.0 \mathrm{a}$ & $5.0 \mathrm{a}$ & $61.1 \mathrm{k}-1$ \\
\hline \multirow[t]{2}{*}{ Oxyfluorfen + oryzalin } & G & 3 & $5.0 \mathrm{a}$ & $5.0 \mathrm{a}$ & $5.0 \mathrm{a}$ & $5.0 \mathrm{a}$ & $5.0 \mathrm{a}$ & $110.0 \mathrm{c}-\mathrm{i}$ \\
\hline & & 6 & $5.0 \mathrm{a}$ & $5.0 \mathrm{a}$ & $5.0 \mathrm{a}$ & $5.0 \mathrm{a}$ & $5.0 \mathrm{a}$ & $113.6 \mathrm{~b}-\mathrm{i}$ \\
\hline \multirow[t]{2}{*}{ Oxyfluorfen + oxadiazon } & G & 3 & $5.0 \mathrm{a}$ & $4.7 \mathrm{ab}$ & $5.0 \mathrm{a}$ & $5.0 \mathrm{a}$ & $5.0 \mathrm{a}$ & 115.9 a-h \\
\hline & & 6 & $5.0 \mathrm{a}$ & $5.0 \mathrm{a}$ & $5.0 \mathrm{a}$ & $5.0 \mathrm{a}$ & $5.0 \mathrm{a}$ & $103.6 \mathrm{~d}-\mathrm{j}$ \\
\hline \multirow{2}{*}{ Oxyfluorfen + pendimethalin } & alin G & 3 & $5.0 \mathrm{a}$ & $5.0 \mathrm{a}$ & $5.0 \mathrm{a}$ & $5.0 \mathrm{a}$ & $5.0 \mathrm{a}$ & $117.6 \mathrm{a}-\mathrm{h}$ \\
\hline & & 6 & $4.9 \mathrm{a}$ & $4.8 a b$ & $5.0 \mathrm{a}$ & $5.0 \mathrm{a}$ & $5.0 \mathrm{a}$ & 129.7 a-e \\
\hline \multirow[t]{2}{*}{ Oxyfluorfen + thiazopyr } & G & 3 & $5.0 \mathrm{a}$ & $5.0 \mathrm{a}$ & $5.0 \mathrm{a}$ & $5.0 \mathrm{a}$ & $5.0 \mathrm{a}$ & $109.4 \mathrm{c}-\mathrm{i}$ \\
\hline & & 6 & $5.0 \mathrm{a}$ & $5.0 \mathrm{a}$ & $5.0 \mathrm{a}$ & $5.0 \mathrm{a}$ & $5.0 \mathrm{a}$ & 129.2 a-e \\
\hline \multirow[t]{2}{*}{ Pendimethalin } & S & 4 & $5.0 \mathrm{a}$ & $5.0 \mathrm{a}$ & $5.0 \mathrm{a}$ & $5.0 \mathrm{a}$ & $5.0 \mathrm{a}$ & 147.7 a \\
\hline & & 8 & $4.9 a$ & $5.0 \mathrm{a}$ & $5.0 \mathrm{a}$ & $5.0 \mathrm{a}$ & $5.0 \mathrm{a}$ & $123.1 \mathrm{a}-\mathrm{g}$ \\
\hline Prodiamine & S & 1.5 & $4.9 \mathrm{a}$ & $5.0 \mathrm{a}$ & $5.0 \mathrm{a}$ & $4.9 \mathrm{a}$ & $5.0 \mathrm{a}$ & $135.9 a-d$ \\
\hline & & 3 & $5.0 \mathrm{a}$ & $5.0 \mathrm{a}$ & $5.0 \mathrm{a}$ & $5.0 \mathrm{a}$ & $5.0 \mathrm{a}$ & 132.6 a-e \\
\hline Simazine & G & 3 & $5.0 \mathrm{a}$ & $5.0 \mathrm{a}$ & $5.0 \mathrm{a}$ & $5.0 \mathrm{a}$ & $5.0 \mathrm{a}$ & $117.7 \mathrm{a}-\mathrm{h}$ \\
\hline & & 6 & $5.0 \mathrm{a}$ & $5.0 \mathrm{a}$ & $5.0 \mathrm{a}$ & $5.0 \mathrm{a}$ & $5.0 \mathrm{a}$ & $110.4 \mathrm{c}-\mathrm{i}$ \\
\hline Thiazopyr & G & 1 & $5.0 \mathrm{a}$ & $5.0 \mathrm{a}$ & $5.0 \mathrm{a}$ & $5.0 \mathrm{a}$ & $5.0 \mathrm{a}$ & $103.9 \mathrm{~d}-\mathrm{j}$ \\
\hline & & 2 & $4.9 \mathrm{a}$ & $4.6 a b$ & $5.0 \mathrm{a}$ & $5.0 \mathrm{a}$ & $5.0 \mathrm{a}$ & $124.3 \mathrm{a}-\mathrm{g}$ \\
\hline Thiazopyr & $\mathrm{S}$ & 1 & $5.0 \mathrm{a}$ & $5.0 \mathrm{a}$ & $5.0 \mathrm{a}$ & $5.0 \mathrm{a}$ & $5.0 \mathrm{a}$ & 130.9 a-e \\
\hline & & 2 & $5.0 \mathrm{a}$ & $5.0 \mathrm{a}$ & $5.0 \mathrm{a}$ & $5.0 \mathrm{a}$ & $5.0 \mathrm{a}$ & $131.1 \mathrm{a}-\mathrm{e}$ \\
\hline Trifluralin & G & 4 & $5.0 \mathrm{a}$ & $5.0 \mathrm{a}$ & $5.0 \mathrm{a}$ & $5.0 \mathrm{a}$ & $5.0 \mathrm{a}$ & $119.2 \mathrm{a}-\mathrm{g}$ \\
\hline & & 8 & $4.9 a$ & $5.0 \mathrm{a}$ & $5.0 \mathrm{a}$ & $5.0 \mathrm{a}$ & $5.0 \mathrm{a}$ & $119.8 \mathrm{a}-\mathrm{g}$ \\
\hline Trifluralin + isoxaben & G & 5 & $5.0 \mathrm{a}$ & $5.0 \mathrm{a}$ & $5.0 \mathrm{a}$ & $5.0 \mathrm{a}$ & $5.0 \mathrm{a}$ & $118.3 a-h$ \\
\hline & & 10 & $4.7 \mathrm{ab}$ & $4.7 \mathrm{ab}$ & $5.0 \mathrm{a}$ & $5.0 \mathrm{a}$ & $5.0 \mathrm{a}$ & $91.6 \mathrm{~g}-\mathrm{j}$ \\
\hline
\end{tabular}

${ }^{\mathrm{z}} \mathrm{G}=$ granules, $\mathrm{S}=$ spray-applied

$\mathrm{y}_{0}=$ dead, 3 = moderate injury, 5 = no injury.

${ }^{\mathrm{X}} \mathrm{M}$ ean separation within columns by the Waller- $\mathrm{D}$ uncan $\mathrm{k}$ ratio method, $\mathrm{k}=100$.

w $1.0 \mathrm{lb} . /$ acre $=1.12 \mathrm{~kg} \cdot \mathrm{ha}^{-1}$.

v28.4 g = $1.0 \mathrm{oz}$.

dichlobenil, which caused relatively minor visible phytotoxicity symptoms at the $1 \times$ rate, and moderate injury at the $2 \times$ rate. Shoot dry mass for dichlobenil-treated areca palms was also significantly less than that of the controls for rates tested. Palms treated with this product had strongly curled rachi and extremely brittle rachi and leaflets. The only other product that caused visibleinjury to areca palms was metolachlor, which resulted in rather minor distortion and tip necrosis of the new growth. Oxyfluorfen, when applied as a spray, resulted in significantly lower shoot dry mass for areca palms, yet this chemical when applied in combination with other chemicals 
T able 3. Phytotoxicity ratings and final shoot dry mass of pygmy date palms treated with 22 preemergent herbicides. Data are means for eight replicate pots.

\begin{tabular}{|c|c|c|c|c|c|c|c|c|}
\hline \multirow[b]{2}{*}{ H erbicide } & \multirow{2}{*}{$\begin{array}{l}\text { pplication } \\
\text { method }^{2}\end{array}$} & \multirow{2}{*}{$\begin{array}{c}\text { R ate } \\
\text { (a.i. lb/acre) }\end{array}$} & \multicolumn{5}{|c|}{ M onths after treatment } & \multirow{2}{*}{$\begin{array}{c}\text { Shoot } \\
\text { dry wt } \\
\text { (g)v }\end{array}$} \\
\hline & & & 2 & 4 & 6 & 8 & 10 & \\
\hline Control & & 0 & $5.0^{y} a^{x}$ & $5.0 \mathrm{a}$ & $5.0 \mathrm{a}$ & $5.0 \mathrm{a}$ & $5.0 \mathrm{a}$ & $145.0 a-f$ \\
\hline \multirow[t]{2}{*}{ Benefin + oryzalin } & G & 6 & $5.0 \mathrm{a}$ & $5.0 \mathrm{a}$ & $5.0 \mathrm{a}$ & $5.0 \mathrm{a}$ & $5.0 \mathrm{a}$ & $106.3 \mathrm{i}-\mathrm{I}$ \\
\hline & & 12 & $5.0 \mathrm{a}$ & $5.0 \mathrm{a}$ & $5.0 \mathrm{a}$ & $5.0 \mathrm{a}$ & $5.0 \mathrm{a}$ & 86.11 \\
\hline \multirow[t]{2}{*}{ DCPA } & $\mathrm{S}$ & 10.5 & $4.9 a b$ & $5.0 \mathrm{a}$ & $5.0 \mathrm{a}$ & $5.0 \mathrm{a}$ & $5.0 \mathrm{a}$ & $117.8 c-1$ \\
\hline & & 21.0 & $5.0 \mathrm{a}$ & $4.9 \mathrm{a}$ & $5.0 \mathrm{a}$ & $5.0 \mathrm{a}$ & $5.0 \mathrm{a}$ & $109.0 \mathrm{~h}-\mathrm{I}$ \\
\hline \multirow[t]{2}{*}{ Dichlobenil } & G & 8 & $4.8 \mathrm{a}-\mathrm{c}$ & $4.7 \mathrm{a}$ & $5.0 \mathrm{a}$ & $5.0 \mathrm{a}$ & $5.0 \mathrm{a}$ & $100.4 \mathrm{k}-\mathrm{I}$ \\
\hline & & 16 & $4.6 \mathrm{c}$ & $5.0 \mathrm{a}$ & $5.0 \mathrm{a}$ & $5.0 \mathrm{a}$ & $5.0 \mathrm{a}$ & $99.5 \mathrm{k}-\mathrm{l}$ \\
\hline \multirow[t]{2}{*}{ Diuron } & $\mathrm{s}$ & 4 & $4.9 a b$ & $5.0 \mathrm{a}$ & $5.0 \mathrm{a}$ & $5.0 \mathrm{a}$ & $5.0 \mathrm{a}$ & $141.2 \mathrm{~b}-\mathrm{h}$ \\
\hline & & 8 & $5.0 \mathrm{a}$ & $5.0 \mathrm{a}$ & $5.0 \mathrm{a}$ & $5.0 \mathrm{a}$ & $5.0 \mathrm{a}$ & $107.8 \mathrm{~h}-\mathrm{I}$ \\
\hline \multirow[t]{2}{*}{ I soxaben } & $\mathrm{s}$ & 1 & $5.0 \mathrm{a}$ & $5.0 \mathrm{a}$ & $5.0 \mathrm{a}$ & $5.0 \mathrm{a}$ & $5.0 \mathrm{a}$ & $119.6 c-1$ \\
\hline & & 2 & $5.0 \mathrm{a}$ & $5.0 \mathrm{a}$ & $5.0 \mathrm{a}$ & $5.0 \mathrm{a}$ & $5.0 \mathrm{a}$ & $111.5 \mathrm{f}-\mathrm{I}$ \\
\hline \multirow[t]{2}{*}{ I soxaben + oryzalin } & $\mathrm{S}$ & 4 & $5.0 \mathrm{a}$ & $5.0 \mathrm{a}$ & $5.0 \mathrm{a}$ & $5.0 \mathrm{a}$ & $5.0 \mathrm{a}$ & $112.0 \mathrm{f}-\mathrm{I}$ \\
\hline & & 8 & $5.0 \mathrm{a}$ & $5.0 \mathrm{a}$ & $5.0 \mathrm{a}$ & $5.0 \mathrm{a}$ & $5.0 \mathrm{a}$ & $103.3 \mathrm{j}-1$ \\
\hline \multirow[t]{2}{*}{ M etolachlor } & $\mathrm{S}$ & 8 & $5.0 \mathrm{a}$ & $5.0 \mathrm{a}$ & $5.0 \mathrm{a}$ & $5.0 \mathrm{a}$ & $5.0 \mathrm{a}$ & $121.1 \mathrm{c}-\mathrm{k}$ \\
\hline & & 16 & $5.0 \mathrm{a}$ & $5.0 \mathrm{a}$ & $4.8 \mathrm{~b}$ & $5.0 \mathrm{a}$ & $5.0 \mathrm{a}$ & $127.9 \mathrm{~b}-\mathrm{k}$ \\
\hline \multirow[t]{2}{*}{ O ryzalin } & $\mathrm{S}$ & 4 & $5.0 \mathrm{a}$ & $5.0 \mathrm{a}$ & $5.0 \mathrm{a}$ & $5.0 \mathrm{a}$ & $5.0 \mathrm{a}$ & $128.6 \mathrm{~b}-\mathrm{k}$ \\
\hline & & 8 & $5.0 \mathrm{a}$ & $5.0 \mathrm{a}$ & $5.0 \mathrm{a}$ & $5.0 \mathrm{a}$ & $5.0 \mathrm{a}$ & $114.3 \mathrm{~d}-\mathrm{I}$ \\
\hline \multirow[t]{2}{*}{ Oxadiazon } & G & 4 & $5.0 \mathrm{a}$ & $5.0 \mathrm{a}$ & $5.0 \mathrm{a}$ & $5.0 \mathrm{a}$ & $5.0 \mathrm{a}$ & $147.0 \mathrm{a}-\mathrm{d}$ \\
\hline & & 8 & $5.0 \mathrm{a}$ & $5.0 \mathrm{a}$ & $5.0 \mathrm{a}$ & $5.0 \mathrm{a}$ & $5.0 \mathrm{a}$ & $127.1 \mathrm{~b}-\mathrm{k}$ \\
\hline \multirow[t]{2}{*}{ Oxadiazon + prodiamine } & G & 2.4 & $5.0 \mathrm{a}$ & $5.0 \mathrm{a}$ & $5.0 \mathrm{a}$ & $5.0 \mathrm{a}$ & $5.0 \mathrm{a}$ & $157.8 \mathrm{ab}$ \\
\hline & & 4.8 & $5.0 \mathrm{a}$ & $5.0 \mathrm{a}$ & $5.0 \mathrm{a}$ & $5.0 \mathrm{a}$ & $5.0 \mathrm{a}$ & 176.6 a \\
\hline \multirow[t]{2}{*}{ O xyflurofen } & $\mathrm{S}$ & 1 & $5.0 \mathrm{a}$ & $5.0 \mathrm{a}$ & $5.0 \mathrm{a}$ & $5.0 \mathrm{a}$ & $5.0 \mathrm{a}$ & $124.8 b-k$ \\
\hline & & 2 & $5.0 \mathrm{a}$ & $5.0 \mathrm{a}$ & $5.0 \mathrm{a}$ & $5.0 \mathrm{a}$ & $5.0 \mathrm{a}$ & $135.2 \mathrm{~b}-\mathrm{j}$ \\
\hline \multirow[t]{2}{*}{ Oxyfluorfen + oryzalin } & G & 3 & $5.0 \mathrm{a}$ & $5.0 \mathrm{a}$ & $5.0 \mathrm{a}$ & $5.0 \mathrm{a}$ & $5.0 \mathrm{a}$ & $124.5 b-k$ \\
\hline & & 6 & $4.9 a b$ & $5.0 \mathrm{a}$ & $5.0 \mathrm{a}$ & $5.0 \mathrm{a}$ & $5.0 \mathrm{a}$ & $117.5 \mathrm{c}-\mathrm{I}$ \\
\hline \multirow[t]{2}{*}{ Oxyfluorfen + oxadiazon } & G & 3 & $5.0 \mathrm{a}$ & $5.0 \mathrm{a}$ & $5.0 \mathrm{a}$ & $5.0 \mathrm{a}$ & $5.0 \mathrm{a}$ & $146.0 \mathrm{a}-\mathrm{e}$ \\
\hline & & 6 & $5.0 \mathrm{a}$ & $4.8 \mathrm{a}$ & $5.0 \mathrm{a}$ & $5.0 \mathrm{a}$ & $5.0 \mathrm{a}$ & $150.1 \mathrm{a}-\mathrm{c}$ \\
\hline \multirow[t]{2}{*}{ O xyfluorfen + pendimethalin } & in $G$ & 3 & $5.0 \mathrm{a}$ & $5.0 \mathrm{a}$ & $5.0 \mathrm{a}$ & $5.0 \mathrm{a}$ & $5.0 \mathrm{a}$ & 129.9 b-k \\
\hline & & 6 & $4.9 a b$ & $5.0 \mathrm{a}$ & $5.0 \mathrm{a}$ & $5.0 \mathrm{a}$ & $5.0 \mathrm{a}$ & 124.6 b-k \\
\hline \multirow[t]{2}{*}{ Oxyfluorfen + thiazopyr } & G & 3 & $5.0 \mathrm{a}$ & $5.0 \mathrm{a}$ & $5.0 \mathrm{a}$ & $5.0 \mathrm{a}$ & $5.0 \mathrm{a}$ & $120.9 c-k$ \\
\hline & & 6 & $5.0 \mathrm{a}$ & $5.0 \mathrm{a}$ & $5.0 \mathrm{a}$ & $5.0 \mathrm{a}$ & $5.0 \mathrm{a}$ & $110.0 \mathrm{~g}-1$ \\
\hline \multirow[t]{2}{*}{ Pendimethalin } & $S$ & 4 & $5.0 \mathrm{a}$ & $5.0 \mathrm{a}$ & $5.0 \mathrm{a}$ & $5.0 \mathrm{a}$ & $5.0 \mathrm{a}$ & $113.9 \mathrm{~d}-1$ \\
\hline & & 8 & $5.0 \mathrm{a}$ & $5.0 \mathrm{a}$ & $5.0 \mathrm{a}$ & $5.0 \mathrm{a}$ & $5.0 \mathrm{a}$ & $104.9 \mathrm{i}-\mathrm{I}$ \\
\hline Prodiamine & $\mathrm{S}$ & 1.5 & $5.0 \mathrm{a}$ & $5.0 \mathrm{a}$ & $5.0 \mathrm{a}$ & $5.0 \mathrm{a}$ & $5.0 \mathrm{a}$ & $125.5 \mathrm{~b}-\mathrm{k}$ \\
\hline & & 3 & $5.0 \mathrm{a}$ & $5.0 \mathrm{a}$ & $5.0 \mathrm{a}$ & $5.0 \mathrm{a}$ & $5.0 \mathrm{a}$ & $128.3 \mathrm{~b}-\mathrm{k}$ \\
\hline Simazine & G & 3 & $5.0 \mathrm{a}$ & $5.0 \mathrm{a}$ & $5.0 \mathrm{a}$ & $5.0 \mathrm{a}$ & $5.0 \mathrm{a}$ & $143.5 \mathrm{a}-\mathrm{g}$ \\
\hline & & 6 & $4.7 \mathrm{bc}$ & $4.9 \mathrm{a}$ & $5.0 \mathrm{a}$ & $5.0 \mathrm{a}$ & $5.0 \mathrm{a}$ & $113.4 \mathrm{~d}-1$ \\
\hline Thiazopyr & G & 1 & $5.0 \mathrm{a}$ & $5.0 \mathrm{a}$ & $5.0 \mathrm{a}$ & $5.0 \mathrm{a}$ & $5.0 \mathrm{a}$ & $108.3 \mathrm{~h}-\mathrm{I}$ \\
\hline & & 2 & $5.0 \mathrm{a}$ & $5.0 \mathrm{a}$ & $5.0 \mathrm{a}$ & $5.0 \mathrm{a}$ & $5.0 \mathrm{a}$ & $110.9 \mathrm{~g}-1$ \\
\hline Thiazopyr & $S$ & 1 & $5.0 \mathrm{a}$ & $5.0 \mathrm{a}$ & $5.0 \mathrm{a}$ & $5.0 \mathrm{a}$ & $5.0 \mathrm{a}$ & $138.1 \mathrm{~b}-\mathrm{i}$ \\
\hline & & 2 & $5.0 \mathrm{a}$ & $5.0 \mathrm{a}$ & $5.0 \mathrm{a}$ & $5.0 \mathrm{a}$ & $5.0 \mathrm{a}$ & $112.9 \mathrm{e}-\mathrm{I}$ \\
\hline Trifluralin & G & 4 & $5.0 \mathrm{a}$ & $5.0 \mathrm{a}$ & $5.0 \mathrm{a}$ & $5.0 \mathrm{a}$ & $5.0 \mathrm{a}$ & $119.5 c-1$ \\
\hline & & 8 & $5.0 \mathrm{a}$ & $5.0 \mathrm{a}$ & $5.0 \mathrm{a}$ & $5.0 \mathrm{a}$ & $5.0 \mathrm{a}$ & $101.8 \mathrm{j}-\mathrm{I}$ \\
\hline Trifluralin + isoxaben & G & 5 & $5.0 \mathrm{a}$ & $5.0 \mathrm{a}$ & $5.0 \mathrm{a}$ & $5.0 \mathrm{a}$ & $5.0 \mathrm{a}$ & $137.8 \mathrm{~b}-\mathrm{i}$ \\
\hline & & 10 & $5.0 \mathrm{a}$ & $5.0 \mathrm{a}$ & $5.0 \mathrm{a}$ & $5.0 \mathrm{a}$ & $5.0 \mathrm{a}$ & $112.2 \mathrm{f}-\mathrm{I}$ \\
\hline
\end{tabular}

${ }^{\mathrm{z}} \mathrm{G}=$ granules, $\mathrm{S}=$ spray-applied.

y $0=$ dead, $3=$ moderate injury, $5=$ no injury.

${ }^{x} \mathrm{M}$ ean separation within columns by the Waller-D uncan $\mathrm{k}$ ratio method, $\mathrm{k}=100$.

${ }^{w} 1.0 \mathrm{lb} /$ acre $=1.12 \mathrm{~kg} \cdot \mathrm{ha}^{-1}$.

v28.4 g = $1.0 \mathrm{oz}$.

in granular form, did not reduce plant growth. Virtually all palmswere symptom-free 10 months after treatment since all foliage present at the time of treatment had been replaced by new leaves by 10 months.
Pygmy date palms were little affected by any of the 22 herbicides tested (Table 3 ). O nly dichlobenil, simazine, and metolachlor at their $2 x$ rates produced any significant damage on this species, and the plants quickly recovered from that. Plant dry weight wassignificantly reduced, however, for many of the products tested. Treatments resulting in significant growth reductions in this species included benefin +oryzalin (both rates), D CPA 
Table 4. Phytotoxicity ratings and final shoot dry mass of mexican fan palms treated with 22 preemergent herbicides. D ata are means for eight replicate pots.

\begin{tabular}{|c|c|c|c|c|c|c|c|c|}
\hline \multirow[b]{2}{*}{ H erbicide } & \multirow{2}{*}{$\begin{array}{l}\text { Application } \\
\text { method }^{2}\end{array}$} & \multirow{2}{*}{$\begin{array}{c}\text { R ate } \\
\text { (a.i. lb/acre) }{ }^{w}\end{array}$} & \multicolumn{5}{|c|}{ M onths after treatment } & \multirow{2}{*}{$\begin{array}{c}\text { Shoot } \\
\text { dry wt } \\
\text { (g) }\end{array}$} \\
\hline & & & 2 & 4 & 6 & 8 & 10 & \\
\hline Control & & 0 & $5.0^{y} a^{x}$ & $5.0 \mathrm{a}$ & $5.0 \mathrm{a}$ & $5.0 \mathrm{a}$ & $5.0 \mathrm{a}$ & $101.4 \mathrm{ab}$ \\
\hline \multirow{2}{*}{ Benefin + oryzalin } & G & 6 & $5.0 \mathrm{a}$ & $5.0 \mathrm{a}$ & $5.0 \mathrm{a}$ & $5.0 \mathrm{a}$ & $5.0 \mathrm{a}$ & $124.9 \mathrm{a}$ \\
\hline & & 12 & $5.0 \mathrm{a}$ & $5.0 \mathrm{a}$ & $5.0 \mathrm{a}$ & $5.0 \mathrm{a}$ & $5.0 \mathrm{a}$ & $80.6 \mathrm{ab}$ \\
\hline \multirow[t]{2}{*}{ DCPA } & $\mathrm{S}$ & 10.5 & $5.0 \mathrm{a}$ & $5.0 \mathrm{a}$ & $4.1 \mathrm{ab}$ & $4.3 \mathrm{a}$ & $3.8 \mathrm{a}$ & $109.8 \mathrm{ab}$ \\
\hline & & 21.0 & $5.0 \mathrm{a}$ & $5.0 \mathrm{a}$ & $5.0 \mathrm{a}$ & $4.3 \mathrm{a}$ & $3.1 \mathrm{a}$ & $92.9 \mathrm{ab}$ \\
\hline \multirow[t]{2}{*}{ Diclobenil } & G & 8 & $4.2 \mathrm{C}$ & $4.6 \mathrm{a}$ & $5.0 \mathrm{a}$ & $5.0 \mathrm{a}$ & $5.0 \mathrm{a}$ & $71.6 \mathrm{ab}$ \\
\hline & & 16 & $3.3 \mathrm{~d}$ & $3.3 \mathrm{c}$ & $3.1 \mathrm{c}$ & $3.1 \mathrm{a}$ & $4.4 \mathrm{a}$ & $38.6 \mathrm{~b}$ \\
\hline \multirow[t]{2}{*}{ Diuron } & $\mathrm{S}$ & 4 & $4.2 \mathrm{C}$ & $5.0 \mathrm{a}$ & $5.0 \mathrm{a}$ & $4.4 \mathrm{a}$ & $4.4 \mathrm{a}$ & $100.4 \mathrm{ab}$ \\
\hline & & 8 & $4.7 \mathrm{~b}$ & $5.0 \mathrm{a}$ & $5.0 \mathrm{a}$ & $4.9 a$ & $3.1 a$ & $95.3 \mathrm{ab}$ \\
\hline \multirow[t]{2}{*}{ I soxaben } & S & 1 & $5.0 \mathrm{a}$ & $5.0 \mathrm{a}$ & $5.0 \mathrm{a}$ & $5.0 \mathrm{a}$ & $5.0 \mathrm{a}$ & $115.4 \mathrm{ab}$ \\
\hline & & 2 & $4.9 a b$ & $5.0 \mathrm{a}$ & $5.0 \mathrm{a}$ & $5.0 \mathrm{a}$ & $5.0 \mathrm{a}$ & $140.3 \mathrm{a}$ \\
\hline \multirow[t]{2}{*}{ I soxaben + oryzalin } & S & 4 & $5.0 \mathrm{a}$ & $5.0 \mathrm{a}$ & $4.1 \mathrm{ab}$ & $5.0 \mathrm{a}$ & $5.0 \mathrm{a}$ & $103.4 \mathrm{ab}$ \\
\hline & & 8 & $5.0 \mathrm{a}$ & $4.4 a b$ & $3.3 b c$ & $4.0 \mathrm{a}$ & $4.4 \mathrm{a}$ & $138.2 \mathrm{a}$ \\
\hline \multirow[t]{2}{*}{ M etolachlor } & $\mathrm{S}$ & 8 & $5.0 \mathrm{a}$ & $4.5 \mathrm{a}$ & $4.7 \mathrm{a}$ & $3.7 \mathrm{a}$ & $3.8 \mathrm{a}$ & $97.5 \mathrm{ab}$ \\
\hline & & 16 & $5.0 \mathrm{a}$ & $3.8 b c$ & $4.2 \mathrm{a}$ & $4.1 \mathrm{a}$ & $3.9 \mathrm{a}$ & $92.9 \mathrm{ab}$ \\
\hline \multirow[t]{2}{*}{ Oryzalin } & S & 4 & $5.0 \mathrm{a}$ & $5.0 \mathrm{a}$ & $4.9 \mathrm{a}$ & $4.6 \mathrm{a}$ & $5.0 \mathrm{a}$ & $102.9 \mathrm{ab}$ \\
\hline & & 8 & $4.9 a b$ & $5.0 \mathrm{a}$ & $5.0 \mathrm{a}$ & $5.0 \mathrm{a}$ & $5.0 \mathrm{a}$ & $91.4 \mathrm{ab}$ \\
\hline \multirow[t]{2}{*}{ Oxadiazon } & G & 4 & $4.9 a b$ & $5.0 \mathrm{a}$ & $5.0 \mathrm{a}$ & $4.7 \mathrm{a}$ & $4.4 \mathrm{a}$ & $98.3 \mathrm{ab}$ \\
\hline & & 8 & $5.0 \mathrm{a}$ & $4.5 \mathrm{a}$ & $4.4 \mathrm{a}$ & $4.4 \mathrm{a}$ & $4.4 \mathrm{a}$ & $113.6 \mathrm{ab}$ \\
\hline \multirow[t]{2}{*}{ Oxadiazon + prodiamine } & G & 2.4 & $5.0 \mathrm{a}$ & $5.0 \mathrm{a}$ & $5.0 \mathrm{a}$ & $5.0 \mathrm{a}$ & $5.0 \mathrm{a}$ & $105.2 \mathrm{ab}$ \\
\hline & & 4.8 & $5.0 \mathrm{a}$ & $5.0 \mathrm{a}$ & $5.0 \mathrm{a}$ & $5.0 \mathrm{a}$ & $5.0 \mathrm{a}$ & $103.0 \mathrm{ab}$ \\
\hline \multirow[t]{2}{*}{ Oxyflurofen } & S & 1 & $5.0 \mathrm{a}$ & $5.0 \mathrm{a}$ & $5.0 \mathrm{a}$ & $4.0 \mathrm{a}$ & $3.8 \mathrm{a}$ & $84.7 \mathrm{ab}$ \\
\hline & & 2 & $5.0 \mathrm{a}$ & $5.0 \mathrm{a}$ & $5.0 \mathrm{a}$ & $4.4 \mathrm{a}$ & $3.8 \mathrm{a}$ & $93.9 \mathrm{ab}$ \\
\hline \multirow[t]{2}{*}{ Oxyfluorfen + oryzalin } & G & 3 & $5.0 \mathrm{a}$ & $5.0 \mathrm{a}$ & $4.4 \mathrm{a}$ & $4.6 \mathrm{a}$ & $3.8 \mathrm{a}$ & $112.6 \mathrm{ab}$ \\
\hline & & 6 & $4.9 a b$ & $5.0 \mathrm{a}$ & $5.0 \mathrm{a}$ & $4.4 \mathrm{a}$ & $3.8 \mathrm{a}$ & $94.6 \mathrm{ab}$ \\
\hline \multirow[t]{2}{*}{ Oxyfluorfen + oxadiazon } & G & 3 & $5.0 \mathrm{a}$ & $5.0 \mathrm{a}$ & $5.0 \mathrm{a}$ & $5.0 \mathrm{a}$ & $5.0 \mathrm{a}$ & $97.8 \mathrm{ab}$ \\
\hline & & 6 & $5.0 \mathrm{a}$ & $5.0 \mathrm{a}$ & $5.0 \mathrm{a}$ & $4.4 \mathrm{a}$ & $4.4 \mathrm{a}$ & $103.6 \mathrm{ab}$ \\
\hline \multirow[t]{2}{*}{ Oxyfluorfen + pendimethali } & alin G & 3 & $5.0 \mathrm{a}$ & $5.0 \mathrm{a}$ & $4.6 \mathrm{a}$ & $4.4 \mathrm{a}$ & $4.4 \mathrm{a}$ & $115.3 \mathrm{ab}$ \\
\hline & & 6 & $5.0 \mathrm{a}$ & $5.0 \mathrm{a}$ & $5.0 \mathrm{a}$ & $5.0 \mathrm{a}$ & $5.0 \mathrm{a}$ & $90.2 \mathrm{ab}$ \\
\hline \multirow[t]{2}{*}{ Oxyfluorfen + thiazopyr } & G & 3 & $5.0 \mathrm{a}$ & $5.0 \mathrm{a}$ & $5.0 \mathrm{a}$ & $5.0 \mathrm{a}$ & $5.0 \mathrm{a}$ & $96.6 \mathrm{ab}$ \\
\hline & & 6 & $5.0 \mathrm{a}$ & $5.0 \mathrm{a}$ & $5.0 \mathrm{a}$ & $5.0 \mathrm{a}$ & $5.0 \mathrm{a}$ & $113.1 \mathrm{ab}$ \\
\hline \multirow[t]{2}{*}{ Pendimethalin } & $\mathrm{S}$ & 4 & $5.0 \mathrm{a}$ & $4.4 a b$ & $4.4 \mathrm{a}$ & $4.0 \mathrm{a}$ & $3.8 \mathrm{a}$ & $109.2 \mathrm{ab}$ \\
\hline & & 8 & $5.0 \mathrm{a}$ & $5.0 \mathrm{a}$ & $4.2 a b$ & $3.8 \mathrm{a}$ & $3.1 \mathrm{a}$ & $88.7 \mathrm{ab}$ \\
\hline Prodiamine & $\mathrm{s}$ & 1.5 & $5.0 \mathrm{a}$ & $5.0 \mathrm{a}$ & $5.0 \mathrm{a}$ & $4.4 \mathrm{a}$ & $4.4 \mathrm{a}$ & 129.6 a \\
\hline & & 3 & $5.0 \mathrm{a}$ & $5.0 \mathrm{a}$ & $5.0 \mathrm{a}$ & $4.4 \mathrm{a}$ & $4.4 \mathrm{a}$ & $155.2 \mathrm{a}$ \\
\hline Simazine & G & 3 & $5.0 \mathrm{a}$ & $5.0 \mathrm{a}$ & $5.0 \mathrm{a}$ & $5.0 \mathrm{a}$ & $5.0 \mathrm{a}$ & $80.2 a b$ \\
\hline & & 6 & $5.0 \mathrm{a}$ & $5.0 \mathrm{a}$ & $5.0 \mathrm{a}$ & $5.0 \mathrm{a}$ & $5.0 \mathrm{a}$ & $100.0 \mathrm{ab}$ \\
\hline Thiazopyr & G & 1 & $5.0 \mathrm{a}$ & $5.0 \mathrm{a}$ & $5.0 \mathrm{a}$ & $5.0 \mathrm{a}$ & $5.0 \mathrm{a}$ & $85.4 a b$ \\
\hline & & 2 & $5.0 \mathrm{a}$ & $5.0 \mathrm{a}$ & $5.0 \mathrm{a}$ & $5.0 \mathrm{a}$ & $4.4 \mathrm{a}$ & $117.7 \mathrm{ab}$ \\
\hline Thiazopyr & $\mathrm{S}$ & 1 & $4.9 a b$ & $5.0 \mathrm{a}$ & $5.0 \mathrm{a}$ & $4.4 \mathrm{a}$ & $4.4 \mathrm{a}$ & $84.8 \mathrm{ab}$ \\
\hline & & 2 & $5.0 \mathrm{a}$ & $5.0 \mathrm{a}$ & $3.1 \mathrm{c}$ & $3.1 \mathrm{a}$ & $3.1 \mathrm{a}$ & $95.6 \mathrm{ab}$ \\
\hline Trifluralin & G & 4 & $5.0 \mathrm{a}$ & $4.9 \mathrm{a}$ & $4.9 \mathrm{a}$ & $5.0 \mathrm{a}$ & $5.0 \mathrm{a}$ & $102.7 \mathrm{ab}$ \\
\hline & & 8 & $5.0 \mathrm{a}$ & $5.0 \mathrm{a}$ & $5.0 \mathrm{a}$ & $5.0 \mathrm{a}$ & $5.0 \mathrm{a}$ & $89.7 \mathrm{ab}$ \\
\hline Trifluralin + isoxaben & G & 5 & $5.0 \mathrm{a}$ & $5.0 \mathrm{a}$ & $5.0 \mathrm{a}$ & $5.0 \mathrm{a}$ & $5.0 \mathrm{a}$ & $99.6 \mathrm{ab}$ \\
\hline & & 10 & $5.0 \mathrm{a}$ & $5.0 \mathrm{a}$ & $5.0 \mathrm{a}$ & $5.0 \mathrm{a}$ & $4.4 \mathrm{a}$ & $96.7 \mathrm{ab}$ \\
\hline
\end{tabular}

${ }^{\mathrm{Z}} \mathrm{G}=$ granules, $\mathrm{S}=$ spray-applied

$\mathrm{y}_{0}=$ dead, 3 = moderate injury, 5 = no injury.

${ }^{\mathrm{x}} \mathrm{M}$ ean separation within columns by the Waller- $\mathrm{D}$ uncan $\mathrm{k}$ ratio method, $\mathrm{k}=100$.

w $1.0 \mathrm{lb} . /$ acre $=1.12 \mathrm{~kg} \cdot \mathrm{ha}^{-1}$.

v28.4 g = $1.0 \mathrm{oz}$.

( $2 \times$ rate), dichlobenil (both rates), diuron $(2 \times$ rate), isoxaben + oryzalin $(2 \times$ rate), oxyfluorfen +thiazopyr ( $2 \times$ rate), pendimethalin $(2 \times$ rate), thiazopyr granules (both rates), and trifluralin ( $2 \times$ rate). Two of the products that stunted pygmy date palm growth contained oryzalin, a chemical that also reduced palm heart yield in field-grown pejibaye (Bactris gasipaes Kunth.) (D eF rank and Clement, 1995). H owever, oryzalin by itself did not result in growth reduction in any of the three species tested in this experiment, suggesting that asynergistic effect of oryzalin with isoxaben and benefin may have occurred on the palms in this study.

$\mathrm{H}$ erbicide injury that occurred 
T able 5. Mean weed count per pot for mexican fan palms treated with 22 preemergent herbicides.

\begin{tabular}{|c|c|c|c|c|c|c|}
\hline \multirow[b]{2}{*}{ H erbicide } & \multirow{2}{*}{$\begin{array}{l}\text { Application } \\
\text { method }^{2}\end{array}$} & \multirow{2}{*}{$\begin{array}{c}\text { R ate } \\
\text { (a.i. Ib/acre) } \\
\end{array}$} & \multicolumn{4}{|c|}{ Months after treatment } \\
\hline & & & 2 & 4 & 6 & 8 \\
\hline \multirow{3}{*}{ Benefin + oryzalin } & & 0 & $1.1 \mathrm{a}^{\mathrm{y}}$ & $6.5 a-c$ & $4.5 \mathrm{a}-\mathrm{c}$ & $1.5 \mathrm{ab}$ \\
\hline & G & 6 & $0.0 \mathrm{~b}$ & $0.0 \mathrm{f}$ & $2.6 b-d$ & $1.3 \mathrm{ab}$ \\
\hline & & 12 & $0.0 \mathrm{~b}$ & $0.4 \mathrm{f}$ & $4.0 \mathrm{a}-\mathrm{d}$ & $2.4 a b$ \\
\hline \multirow[t]{2}{*}{ DCPA } & $\mathrm{s}$ & 10.5 & $0.5 \mathrm{~b}$ & $6.5 a-c$ & $0.9 \mathrm{~cd}$ & $0.1 \mathrm{~b}$ \\
\hline & & 21.0 & $0.1 \mathrm{~b}$ & $7.6 \mathrm{ab}$ & $5.0 \mathrm{ab}$ & $2.8 \mathrm{ab}$ \\
\hline \multirow[t]{2}{*}{ Dichlobenil } & G & 8 & $0.0 \mathrm{~b}$ & $0.0 \mathrm{f}$ & $1.6 \mathrm{~b}-\mathrm{d}$ & $1.1 \mathrm{ab}$ \\
\hline & & 16 & $0.0 \mathrm{~b}$ & $1.1 \mathrm{f}$ & $2.5 b-d$ & $2.5 \mathrm{ab}$ \\
\hline \multirow[t]{2}{*}{ Diuron } & $\mathrm{S}$ & 4 & $0.6 \mathrm{~b}$ & $4.1 \mathrm{~b}-\mathrm{f}$ & $1.6 \mathrm{~b}-\mathrm{d}$ & $0.5 \mathrm{~b}$ \\
\hline & & 8 & $0.0 \mathrm{~b}$ & $3.9 b-f$ & $3.5 a-d$ & $1.5 \mathrm{ab}$ \\
\hline \multirow[t]{2}{*}{ I soxaben } & $\mathrm{S}$ & 1 & $1.6 \mathrm{a}$ & $8.6 a$ & $2.0 \mathrm{~b}-\mathrm{d}$ & $2.4 \mathrm{ab}$ \\
\hline & & 2 & $0.5 \mathrm{~b}$ & $5.6 a-e$ & $7.3 \mathrm{a}$ & $0.9 \mathrm{~b}$ \\
\hline \multirow[t]{2}{*}{ I soxaben + oryzalin } & $\mathrm{s}$ & 4 & $0.0 \mathrm{~b}$ & $0.0 \mathrm{f}$ & $1.1 \mathrm{~b}-\mathrm{d}$ & $1.1 \mathrm{ab}$ \\
\hline & & 8 & $0.0 \mathrm{~b}$ & $0.0 \mathrm{f}$ & $1.1 \mathrm{~b}-\mathrm{d}$ & $0.1 \mathrm{~b}$ \\
\hline \multirow[t]{2}{*}{ M etolachlor } & $\mathrm{s}$ & 8 & $0.0 \mathrm{~b}$ & $3.8 \mathrm{~b}-\mathrm{f}$ & $1.5 \mathrm{~b}-\mathrm{d}$ & $0.0 \mathrm{~b}$ \\
\hline & & 16 & $0.0 \mathrm{~b}$ & $3.0 c-f$ & $3.1 b-d$ & $0.8 \mathrm{~b}$ \\
\hline \multirow[t]{2}{*}{ O ryzalin } & $\mathrm{s}$ & 4 & $0.0 \mathrm{~b}$ & $6.5 a-c$ & $3.1 b-d$ & $1.0 \mathrm{ab}$ \\
\hline & & 8 & $0.0 \mathrm{~b}$ & $6.0 a-d$ & $1.0 \mathrm{~b}-\mathrm{d}$ & $0.1 \mathrm{~b}$ \\
\hline \multirow[t]{2}{*}{ Oxadiazon } & G & 4 & $0.0 \mathrm{~b}$ & $0.4 \mathrm{f}$ & $2.6 b-d$ & $0.9 \mathrm{~b}$ \\
\hline & & 8 & $0.0 \mathrm{~b}$ & $0.0 \mathrm{f}$ & $0.5 \mathrm{~cd}$ & $0.5 \mathrm{~b}$ \\
\hline \multirow[t]{2}{*}{ Oxadiazon + prodiamine } & G & 2.4 & $0.0 \mathrm{~b}$ & $0.0 \mathrm{f}$ & $0.4 \mathrm{~d}$ & $1.8 \mathrm{ab}$ \\
\hline & & 4.8 & $0.0 \mathrm{~b}$ & $0.0 \mathrm{f}$ & $0.8 \mathrm{~cd}$ & $0.5 \mathrm{~b}$ \\
\hline \multirow[t]{2}{*}{ O xyflurofen } & $\mathrm{s}$ & 1 & $0.0 \mathrm{~b}$ & $2.1 c-f$ & $1.8 b-d$ & $1.6 \mathrm{ab}$ \\
\hline & & 2 & $0.0 \mathrm{~b}$ & $1.4 \mathrm{ef}$ & $2.8 b-d$ & $0.5 \mathrm{~b}$ \\
\hline \multirow[t]{2}{*}{ Oxyfluorfen + oryzalin } & G & 3 & $0.0 \mathrm{~b}$ & $0.0 \mathrm{f}$ & $0.4 \mathrm{~d}$ & $0.6 \mathrm{~b}$ \\
\hline & & 6 & $0.0 \mathrm{~b}$ & $0.0 \mathrm{f}$ & $0.0 \mathrm{~d}$ & $0.0 \mathrm{~b}$ \\
\hline \multirow[t]{2}{*}{ Oxyfluorfen + oxadiazon } & G & 3 & $0.0 \mathrm{~b}$ & $0.0 \mathrm{f}$ & $0.1 \mathrm{~d}$ & $0.1 \mathrm{~b}$ \\
\hline & & 6 & $0.0 \mathrm{~b}$ & $0.0 \mathrm{f}$ & $0.0 \mathrm{~d}$ & $0.0 \mathrm{~b}$ \\
\hline \multirow[t]{2}{*}{ Oxyfluorfen + pendimethalin } & G & 3 & $0.0 \mathrm{~b}$ & $0.0 \mathrm{f}$ & $0.5 \mathrm{~cd}$ & $0.1 \mathrm{~b}$ \\
\hline & & 6 & $0.0 \mathrm{~b}$ & $0.0 \mathrm{f}$ & $0.0 \mathrm{~d}$ & $0.0 \mathrm{~b}$ \\
\hline \multirow[t]{2}{*}{ O xyfluorfen + thiazopyr } & G & 3 & $0.0 \mathrm{~b}$ & $0.0 \mathrm{f}$ & $0.0 \mathrm{~d}$ & $0.0 \mathrm{~b}$ \\
\hline & & 6 & $0.0 \mathrm{~b}$ & $0.0 \mathrm{f}$ & $0.4 \mathrm{~d}$ & $0.0 \mathrm{~b}$ \\
\hline \multirow[t]{2}{*}{ Pendimethalin } & $\mathrm{s}$ & 4 & $0.0 \mathrm{~b}$ & $1.1 \mathrm{f}$ & $3.3 a-d$ & $1.3 \mathrm{ab}$ \\
\hline & & 8 & $0.0 \mathrm{~b}$ & $1.5 \mathrm{ef}$ & $1.0 \mathrm{~b}-\mathrm{d}$ & $0.0 \mathrm{~b}$ \\
\hline \multirow[t]{2}{*}{ Prodiamine } & $\mathrm{S}$ & 1.5 & $0.0 \mathrm{~b}$ & $2.6 c-f$ & $2.8 b-d$ & $0.4 \mathrm{~b}$ \\
\hline & & 3 & $0.0 \mathrm{~b}$ & $1.3 \mathrm{ef}$ & $2.8 b-d$ & $1.4 \mathrm{ab}$ \\
\hline \multirow{2}{*}{ Simazine } & G & 3 & $0.0 \mathrm{~b}$ & $0.5 \mathrm{f}$ & $5.0 \mathrm{ab}$ & $4.5 \mathrm{a}$ \\
\hline & & 6 & $0.0 \mathrm{~b}$ & $0.5 \mathrm{f}$ & $3.4 a-d$ & $1.4 \mathrm{ab}$ \\
\hline Thiazopyr & G & 1 & $0.0 \mathrm{~b}$ & $0.0 \mathrm{f}$ & $2.6 b-d$ & $1.3 \mathrm{ab}$ \\
\hline & & 2 & $0.0 \mathrm{~b}$ & $0.0 \mathrm{f}$ & $1.0 \mathrm{~b}-\mathrm{d}$ & $0.8 \mathrm{~b}$ \\
\hline Thiazopyr & $\mathrm{s}$ & 1 & $0.1 \mathrm{~b}$ & $1.8 \mathrm{~d}-\mathrm{f}$ & $1.5 b-d$ & $0.8 \mathrm{~b}$ \\
\hline & & 2 & $0.0 \mathrm{~b}$ & $1.3 \mathrm{ef}$ & $0.6 \mathrm{~cd}$ & $0.0 \mathrm{~b}$ \\
\hline Trifluralin & G & 4 & $0.0 \mathrm{~b}$ & $0.0 \mathrm{f}$ & $1.3 b-d$ & $1.3 \mathrm{ab}$ \\
\hline & & 8 & $0.0 \mathrm{~b}$ & $0.0 \mathrm{f}$ & $1.4 b-d$ & $1.6 \mathrm{ab}$ \\
\hline Trifluralin + isoxaben & G & 5 & $0.0 \mathrm{~b}$ & $0.1 \mathrm{f}$ & $2.8 b-d$ & $0.9 \mathrm{~b}$ \\
\hline & & 10 & $0.0 \mathrm{~b}$ & $0.4 \mathrm{f}$ & $2.0 b-d$ & $3.5 \mathrm{ab}$ \\
\hline
\end{tabular}

${ }^{\mathrm{z}} \mathrm{G}=$ granules, $\mathrm{S}=$ spray-applied.

yM ean separation within columns by the Waller-D uncan $\mathrm{k}$ ratio method, $\mathrm{k}=100$.

x $1.0 \mathrm{lb} . /$ acre $=1.12 \mathrm{~kg} \cdot \mathrm{ha}^{-1}$.

during thefirst two monthson mexican fan palms typically appeared as tip necrosis and/ or distortion of the new leaves. Injury that occurred after 2 months generally affected the apical meristem and usually resulted in death of the spear leaf, followed by death of the entire palm. The latter symptoms were similar in appearance to several bud rot diseases that may have affected palms stressed by some of the herbicides. Relatively few treatments were completely free from the latter type of injury. However, since none of the control palms exhibited bud rot symptoms, any bud rotting agents involved were probably secondary invaders following bud injury by the various herbicides. Those products that did not injure mexican fan palms at either rate included benefin +oryzalin, oxadiazon +prodiamine, oxyfluorfen +thiazopyr, and simazine (T able 4). In addition, the $1 \times$ rates of isoxaben, oxyfluorfen + oxadiazon, thiazopyr granules, trifluralin + isoxaben and the $2 \times$ rate of trifluralin were also free of symptoms throughout the experiment. H owever, due to the considerable variability in the phytotoxicity ratings for this spe- 
cies, only dichlobenil (both rates), diuron (both rates), isoxaben + oryzalin ( $2 \times$ rate), and metolachlor ( $2 \times$ rate) ever had phytotoxicity ratings significantly different from thecontrols. Shoot dry mass did not differ significantly among treatments.

The only product that was consistently phytotoxic to all three species of palms was dichlobenil. M etolachlor resulted in minor phytotoxicity in areca palm and mexican fan palm in thisstudy, but $M$ eerow and Broschat (1991) reported severe phytotoxicity from this product on similar and additional palm species. The most likely explanation for this difference was that this product was immediately rinsed off the foliage following application in this study as recommended on the label. M eerow and Broschat (1991) did not rinse the foliagefollowing application in their study.

The products containing oxyfluorfen +oryzalin and oxyfluorfen + pendimethalin wereevaluated on four species of palms, including areca palm, by Donselman and Broschat (1986) who found these productsto be moderately phytotoxic on all four species. Although these products may have been changed by their manufacturers during the intervening 14 years, it is also possible that environmental factors may influence their activity and safety. $O$ xadiazon was quite safe on the five species tested by Donselman and Broschat (1986) and N eel (1977) and was safe on areca palms and pygmy date palmsin thisstudy aswell. I t wasslightly injurious to mexican fan palms in this study, however.

WeEd control. The most important weed species (in order of occurrencefrequency) wereartilleryfern [Pilea microphylla (L.) Liebm.], watersnake hemp (Fatoua villosa Gaud.), hairy bittercress (Cardamine hirsuta L.), spurges (Euphorbia L. sp.), longstalked phyllanthus(PhyllanthustenellusR oxb.), asiatic hawksbeard [Y oungia japonica (L.) D C.], creeping woodsorrel (Oxalis corniculata L.), common beggartick [Bidens alba (L.) DC.], boston fern [N ephrolepisexaltata (L.) Schott.], and florida pellatory (Parietaria floridana $\mathrm{N}$ utt.). N umbers of each individual weed species were generally too low to show significant differencesamong treatments with respect to their control and total weed count per pot was therefore used for statistical analysis. Sinceoverall weed infestations were most severe in the mexican fan palms, only weed control data for that species was presented.

All 22 products except isoxaben ( $1 \times$ rate) gave significantly better weed control than the untreated control during the first 2 months (Table 5). At 4 months, DCPA (both rates), diuron (both rates), isoxaben (both rates), metolachlor (both rates), oryzalin (both rates), oxyfluorfen ( $1 \times$ rate), and prodiamine ( $1 \times$ rate) provided no significant weed control. All of these productsareapplied asaspray. After 6 months, only oxadiazon + prodiamine ( $1 \times$ rate), oxyfluorfen + oryzalin (both rates), oxyfluorfen + oxadiazon (both rates), oxyfluorfen + pendimethalin ( $2 \times$ rate), and oxyfluorfen +thiazopyr (both rates) provided significant weed control. All of these products are granular formulations. By 8 months, there were no significant differences between treatments and the controls.

\section{Conclusions}

With the exceptions of dichlobenil and metolachlor, which caused some degree of injury on all three palm species, most of the products tested were relatively safefor useon thesecontainergrown palmswhen applied according to their label and at twice the labeled rate. Although visible injury was relatively rare on pygmy date palms, many of the products did result in reduced growth rates in that species. Virtually all of the 22 herbicide treatments provided good weed control for 2 months, but most of the spray-applied formulationswere ineffective by 4 months. Granular products generally lasted longer, with those containing oxyfluorfen plus another activeingredient lasting at least 6 months.

\section{Literature cited}

D eFrank, J and C.R. Clement. 1995. Weed control in pejibaye heart of palm plantations in $\mathrm{H}$ awaii. $\mathrm{H}$ ortScience 30:12151216.

Donselman, H . and T.K. Broschat. 1986. Phytotoxicity of several pre- and postemergent herbicides on container grown palms. Proc. Fla. St. Hort. Soc. 99:273-274.

M eerow, A.W. and T.K. Broschat. 1991. Phytotoxicity of the pre-emergent herbicide metolachlor on containerized palms. Foliage Dig. 17(9):6.

N eel, P.L. 1977. Effects of oxadiazon preemergence herbicide on weed control and growth of sixteen species of containerized ornamental plants. Proc. Fla. St. Hort. Soc. 90:353-355. 